\title{
To staple or not to staple the symptomatic rectocele
}

\author{
P.-A. Lehur ${ }^{1}$ (D) B. Pravini ${ }^{1} \cdot$ D. Christoforidis ${ }^{1}$
}

Received: 7 April 2019 / Accepted: 2 December 2019 / Published online: 9 December 2019

(c) Springer Nature Switzerland AG 2019

See: "Giarratano et al., Stapled transanal rectal resection for the treatment of rectocele associated with obstructed defecation disorder: a large series of 262 consecutive patients" and "Regadas et al., Impact of TRREMS procedure on symptoms of obstructed defecation due to rectocele: Predictive factors and outcome".

In the last 2 years, two publications showed, rather convincingly, the superiority of laparoscopic ventral mesh rectopexy (LVMR) over stapled transanal rectal resection (STARR) in the management of obstructed defecation syndrome (ODS) associated with pelvic structural abnormalities, such as high-grade rectal intussusception and/or large rectocele $[1,2]$. These papers confirmed the empirical trend in several European referral centers for decreased use of transanal stapling procedures of any type for this frequent and complex condition that intermingles functional and anatomical disorders [3].

This month and earlier this year in Techniques in Coloproctology, two teams, one from Italy and the other from Brazil, are renewing interest in stapling procedures for symptomatic rectoceles by reporting their personal results either with the original stapled transanal rectal resection (STARR) procedure or the transanal repair of rectocele and rectal mucosectomy with a single circular stapler (TRREMS) $[4,5]$.

Thus, the debate initiated some 15-20 years ago on how we should treat a patient presenting with ODS with an associated symptomatic rectocele still ongoing. In this editorial, we would like to propose some suggestions on how to select one or other surgical approach. Then, on a more general basis, we will discuss the requirements for an ethical, meaningful and valid research in functional coloproctology, in 2020 [6].

P.-A. Lehur

dr.pa.lehur@gmail.com

1 Coloproctology Unit, Ospedale Regionale di Lugano, 6900 Lugano, Switzerland
Not long ago, colorectal surgeons focused interest on rectocele and ODS management. Functional anorectal and pelvic floor disorders, now a specific and rather complex field in proctology, are now better understood using the current tools at our disposal: defecography (which can be performed with conventional radiology, with magnetic resonance imaging (MRI) (that we favor) or even echographically [5]), and anal physiology tests. Thanks to a better understanding of the underlying pathophysiology, new dedicated "colorectal" procedures have been designed as an alternative approach to the traditional long-known gynecological procedures. They include either an abdominal "conservative" (today predominantly laparoscopic), mesh rectal suspension or a transanal "resectional", predominantly stapled, endorectal procedure.

\section{Some tips on how to select a surgical option to treat ODS and/or rectocele}

It is not always easy to decide between an abdominal or perineal approach to treat these generally associated conditions; but in our view, there are situations where choosing an abdominal laparoscopic approach should be clear. This is the case for those patients who require a global pelvic reconstruction including both an anterior suspension and a LVMR. This involves a thorough preoperative clinical and radiological pelvi-perineal examination, and the contribution of a urogynecologist, ideally with a traceable decision made at a pelvic floor multidisciplinary team meeting. It would have been of interest to know in the series presented by Giarratano et al. and Regadas et al. [4, 5] if and how many patients were excluded from the transanal approach for this rather common scenario. Second, for patients already incontinent or at risk of fecal incontinence, it is difficult to predict what the consequences will be of the anal dilation required for inserting the stapler into the rectum and of the resection itself that reduces rather significantly the volume/capacity of the rectal ampulla. Wisely, in both studies, patients with sphincter defects were excluded, but in this population of patients in their $50 \mathrm{~s}$ who often suffer sequelae 
of childbirth injuries and pelvic floor descent, a normal anal sphincter is rarely seen. Furthermore, it has to be kept in mind that "post-stapling" fecal incontinence of any degree is extremely difficult to solve. Finally, patients presenting with an associated pelvic pain syndrome require special attention before they are offered a transanal/transperineal procedure. Increased pain, the exact mechanisms of which are unclear although probably linked to the postoperative inflammatory reaction, may occur after stapled procedure and is often severe and difficult to treat. Another point of caution regards the presence of an enterocele, frequently associated with rectocele and rectal intussusception. This Douglas pouch "hernia" (peritoneocele) is not easy to recognize clinically and a radiological investigation is mandatory. Even if there is no clear recommendation to avoid stapled procedures in these usually large-volume entero-/rectoceles, the correction of the hernia is conceptually less convincing with transanal stapling than with LVMR. More importantly, the risk of incorporating the peritoneal sac and eventually its content (small bowel loops or sigmoid) into the staple line should be kept in mind. Fortunately, no complication of that sort is reported in the two publications $[4,5]$.

Thus, aside from the above-mentioned strong indications for LVMR, there are a number of patients in a gray zone where deciding between a transanal resectional procedure or an abdominal rectal suspension is difficult, in the absence of valid recommendations. We must confess that in our experience and nowadays, more patients were found to fit in the LVMR category rather than in the STARR category. Giarratano et al. and Regadas et al. should be congratulated for recruiting such a large number of patients for transanal stapling and for so few patients having been lost to followup $[4,5]$.

\section{An unsolved debate and the urgent need for validated guidance}

Colorectal surgeons have been extraordinarily ingenious in their approach to anorectal functional disorders, a new field in our specialty. For the condition we are interested in here, many innovative proposals with their variants, either to suspend or to resect and staple have been designed. Doubtless innovation is key to surgery. Nevertheless, it is of utmost importance to submit a new technology (procedure) to a carefully structured validation process before introducing it into the routine clinical practice. It is nowadays especially true that we need evidence-based guidance for the management of ODS and/or rectocele and rectal intussusception due to lack of well-conducted studies [6]. We fully recognize the difficulty in setting up randomized controlled trials (RCTs) to obtain such evidence as surgical expertise in these procedures differs, patients' acceptance of randomization varies and the surgeon's belief in one or the other option removes the equipoise necessary for randomisation. Solid evidence could probably be obtained from registries or cohort studies provided that suitable data are reliably recorded and thoroughly reported.

There are requirements for the way we perform research in this field that can be considered as universally accepted and do not need further discussion: reporting past medical and obstetric history (childbirth/hysterectomy) to identify their impact on outcome; use of validated standardized grading systems for clinical and radiological findings to allow for comparison of patients; use of conservative medical treatment and pelvic floor retraining prior to surgical indication; and reporting of well-identified specific complications. Our objective is to focus on what could provide evidence for or against a specific procedure in terms of bowel and pelvic symptoms and on bowel and pelvic related quality of life (QoL) or said differently "comfort". Validated scoring systems, simple to use on digital platforms, are now available and should be used [7]. They give more information on patients' global pelvic health including all functions and can be analyzed independently if needed, always in comparison with baseline prospectively recorded data. They would detect for instance some dissatisfaction if, following a successful transanal procedure, a patient has to be operated on again for a cystohysterocele few months later.

"Patient-reported outcome measures" (PROMs) are today mainstream for the evaluation of results of procedures intended to improve QoL. There is an urgent need to develop and validate them for ODS and rectocele surgery of any kind. They could be based on a model similar to the International Consultation on Incontinence Questionnaire-B (ICIQB) for fecal incontinence, eventually in its short form which is easier to use [8]. The level of patient satisfaction with the operative result would be described much better than in two series discussed above, where a three-grade scale (excellent, good or poor) was used. With a "poor" outcome noted only in $1 \%$ [4] and $2.5 \%$ [5] at 1 year, respectively, these results are rather impressive considering the complexity of the treated population in its peri-menopausal age usually presenting with associated psychological symptoms. However, the authors do not state how the satisfaction outcomes were collected: who did it and how was it done-phone or physical interview, anonymous questionnaires or not, with or without clinical examination? These are all factors known to affect the reliability of results. Finally, to support the above comments, it is worth citing another recent publication on the long-term results of STARR [9]. Sixty, out of 74 (81\%) patients who had STARR, answered a questionnaire at 10-year follow-up: $38 \%$ reported persistent perineal pain, $22 \%$ complained of defecation urgency, while recurrence of ODS symptoms occurred in $40 \%$. Only $35 \%$ of the patients 
who responded reported a high degree of satisfaction with the operation.

It seems that the time has come to proceed differently when dealing with studies on functional pelvic floor disorders. This is an ethical and pragmatic issue if we want to continue serving our patients and our surgical community. Ethically conducted surgical innovation in functional anorectal disorders must be encouraged. It is of upmost importance that reporting of results follows the rigorous rules of scientific objectivity. This is the only way we will be able to build reliable evidence in this area.

\section{Funding None.}

\section{Compliance with ethical standards}

Conflict of interest Paul-Antoine Lehur is a former consultant for American Medical Systems and Torax Medical. He is currently a consultant for Medtronic SA and B. Braun.

\section{References}

1. Altomare DF, Picciariello A, Memeo R et al (2018) Pelvic floor function following ventral rectopexy versus STARR in the treatment of obstructed defecation. Tech Coloproctol 22:289-294

2. Madbouly KM, Mohii AD (2019) Laparoscopic ventral rectopexy versus stapled transanal rectal resection for treatment of obstructed defecation in the elderly: long-term results of a prospective randomized study. Dis Colon Rectum 62:47-55
3. Kim M, Meurette G, Ragu R, Lehur PA (2016) Current surgical treatment of obstructed defecation among selected European opinion leaders in pelvic floor surgery. Tech Coloproctol 20:395-399

4. Giarratano G, Toscana C, Toscana E et al (2019) Stapled transanal rectal resection for the treatment of rectocele associated with obstructed defecation disorder: a large series of 262 consecutive patients. Tech Coloproctol 23:231-237. https://doi.org/10.1007/ s10151-019-01944-9

5. Regadas FS, Regadas SM, Rodrigues LV et al (2019) Impact of TRREMS on symptoms of obstructed defecation due to rectocele: predictive factors and outcomes. Tech Coloproctol. https://doi. org/10.1007/s10151-019-02131-6

6. Van Geluwe B, Stuto A, Da Pozzo F et al (2014) Relief of obstructed defecation syndrome after stapled transanal rectal resection (STARR): a meta-analysis. Acta Chir Belg 114:189-197

7. Altomare DF, Di Lena M, Giuratrabocchetta $S$ et al (2014) The three axial perineal evaluation (TAPE) score: a new scoring system for comprehensive evaluation of pelvic floor function. Colorectal Dis 16:459-468

8. Cotterill N, Norton C, Avery KN et al (2011) Psychometric evaluation of a new patient-completed questionnaire for evaluating anal incontinence symptoms and impact on quality of life: the ICIQ-B. Dis Colon Rectum 54:1235-1250

9. Sciano di Visconte M, Nicolì F, Pasquali A et al (2018) Clinical outcomes of stapled transanal rectal resection for obstructed defaecation syndrome at 10-year follow-up. Colorectal Dis 20:614-622

Publisher's Note Springer Nature remains neutral with regard to jurisdictional claims in published maps and institutional affiliations. 